\title{
Characterization of deep impurities in semiconductors by terahertz tunneling ionization
}

\author{
E. Ziemann, S. D. Ganichev, ${ }^{\text {a) }}$ and W. Prettl \\ Institut für Experimentelle und Angewandte Physik, Universität Regensburg, 93040 Regensburg, Germany \\ I. N. Yassievich and V. I. Perel \\ A. F. Ioffe Physicotechnical Institute, Russian Academy of Sciences, 194021 St. Petersburg, Russia
}

(Received 9 December 1999; accepted for publication 11 January 2000)

\begin{abstract}
Tunneling ionization in high frequency fields as well as in static fields is suggested as a method for the characterization of deep impurities in semiconductors. It is shown that an analysis of the field and temperature dependences of the ionization probability allows to obtain defect parameters like the charge of the impurity, tunneling times, the Huang-Rhys parameter, the difference between optical and thermal binding energy, and the basic structure of the defect adiabatic potentials. Compared to static fields, high frequency electric fields in the terahertz-range offer various advantages, as they can be applied contactlessly and homogeneously even to bulk samples using the intense radiation of a high power pulsed far-infrared laser. Furthermore, impurity ionization with terahertz radiation can be detected as photoconductive signal with a very high sensitivity in a wide range of electric field strengths. (C) 2000 American Institute of Physics. [S0021-8979(00)04508-4]
\end{abstract}

\section{INTRODUCTION}

Investigation of the effect of an electric field on thermal ionization and trapping of carriers has been widely used to probe deep impurities in semiconductors. The standard method for the investigation of deep centers, in particular, the determination of the ionization energy, is deep level transient spectroscopy (DLTS) ${ }^{1}$ which is applied in various modifications.

The application of strong electric fields to semiconductors with deep centers leads to the stimulation of ionization/ capture processes due to the Poole-Frenkel effect, phonon assisted tunneling, and direct tunneling. The Poole-Frenkel effect occurs for charged impurities only and can be observed for relatively small electric field strengths, whereas all types of impurities can be ionized by tunneling.

The theory of phonon assisted tunneling in static electric fields was first considered quantum mechanically. MakramEbeid and Lannoo carried out the most extensive numerical studies. ${ }^{2}$ A semiclassical theory of phonon assisted tunneling was presented by Karpus and Perel $^{3}$ yielding analytical solutions of the problem, which we will use here. Both theoretical approaches are in good agreement. Phonon assisted tunneling has been observed for a number of deep impurities in static electric fields ${ }^{4,5}$ as well as in high frequency electric fields. ${ }^{6-8}$

Here, we will show that the investigation of phonon assisted tunneling in strong static electric or alternating fields, in particular of terahertz frequencies, can be used to obtain the parameters of multiphonon transitions determining the nonradiative recombination rate. The multiphonon parameters of deep-level impurities, the structure of their adiabatic

\footnotetext{
a) Also at A. F. Ioffe Physicotechnical Institute, Russian Academy of Sciences, 194021 St. Petersburg, Russia;

electronic mail: sergey.ganichev@physik.uni-regensburg.de
}

potentials, and the recombination kinetic of nonequilibrium carriers can be obtained from the field and temperature dependences of phonon assisted tunneling ionization. The application of high frequency fields in the terahertz range gives two important advantages as compared to static fields. High terahertz electric fields can be applied in a contactless manner to any kind of bulk or structured semiconductor samples using the intense radiation of a high-power pulsed farinfrared laser at photon energies being much smaller than the binding energy of the impurities. Furthermore, the ionization probability may easily be determined by detecting photoconductivity offering a very sensitive method of high dynamic range. By this technique, the electric field dependence of the ionization probability can be measured over several orders of magnitude of the electric field strength.

\section{TUNNELING IONIZATION IN ALTERNATING ELECTRIC FIELDS}

Thermal emission of carriers from impurity bound states into the continuum at zero electric field is usually accomplished by thermal activation of the system in the adiabatic bound state potential and tunneling of the defect from the bound configuration into the ionized configuration. This defect tunneling process is illustrated in Fig. 1. In the semiclassical limit, the tunneling trajectory can be split into two parts, one below the adiabatic potential of the bound state $U_{1}$, the other below that of the ionized state $U_{2} \cdot{ }^{3}$ The partial tunneling processes under $U_{1}$ and $U_{2}$ are characterized by Büttiker-Landauer tunneling times ${ }^{9} \tau_{1}$ and $\tau_{2}$, respectively.

An electric field causes electron tunneling through the barrier formed by the impurity potential and the potential of the electric field, as shown by the solid line trajectory in Fig. 2. Hence, the electron leaves the impurity at an energy $\varepsilon$ below the lower edge of the continuum. This is taken into account in the configuration coordinate model by shifting the 


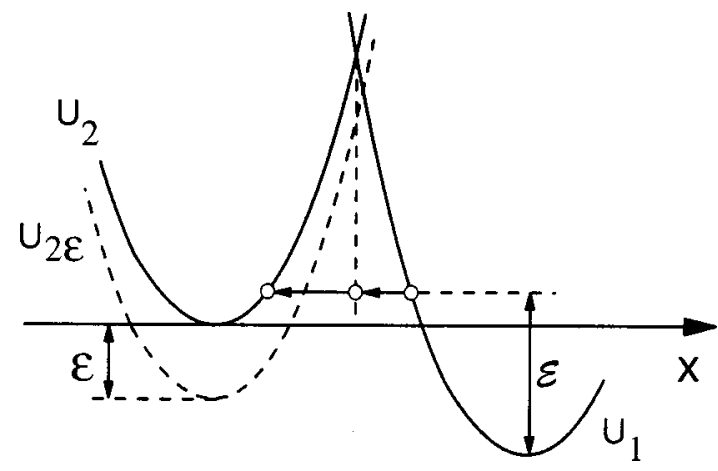

FIG. 1. Adiabatic potential configurations as used for $D X$ centers. The energy of the vibrating defect and the electron is shown as a function of a configuration coordinate $x$ for the electron bound to $\left(U_{1}\right)$ and detached from $\left(U_{2}\right)$ the defect at energy of electron $\varepsilon=0$. The potential plotted in broken lines corresponds to an electron with negative kinetic energy $-\varepsilon$ which left the impurity due to tunneling in an external electric fields (see Fig. 2).

potential of the ionized state $U_{2}$ by $\varepsilon$ to $U_{2 \varepsilon}$ due to the lowering of the total energy of electron and ionized impurity. The tunneling barrier gets thinner causing enhancement of ionization. ${ }^{5}$ Thus, ionization of deep impurities in external electric fields is accomplished by two simultaneous tunneling processes, namely, electron tunneling and defect tunneling. In semiclassical approximation, the probability of the ionization process may be written as

$$
e(E)=\iint P_{e}(\varepsilon) P_{d}(\mathcal{E}) \exp \left(-\mathcal{E} / k_{B} T\right) d \varepsilon d \mathcal{E},
$$

where $P_{e}$ and $P_{d}$ are the electron and the defect tunneling probabilities, respectively, $T$ is the temperature, $\varepsilon$ the electron energy at tunneling, and $\mathcal{E}$ the corresponding defect tunneling energy. The Boltzmann factor takes into account the thermal excitation of the system in the adiabatic bound state potential ( $U_{1}$ in Fig. 1).

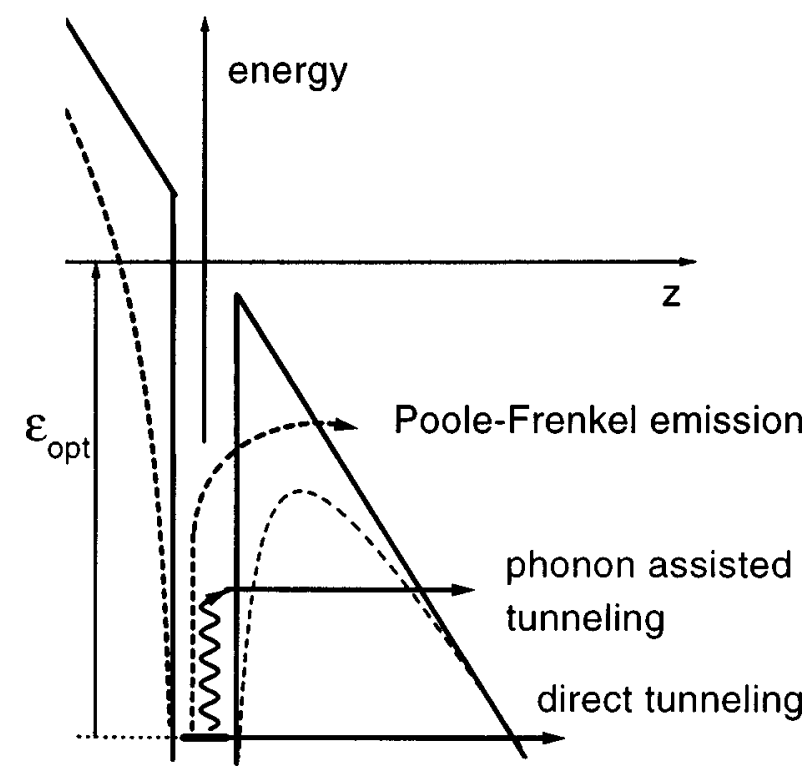

FIG. 2. Deep impurity potential binding the electron in an electric field, solid line for neutral impurity, dashed line for charged attractive impurity. The arrows show the semiclassical trajectories for different ionization processes.
As long as the energy of the tunneling electron $\varepsilon$ is smaller than the defect tunneling energy $\mathcal{E}$ and the thermal binding energy $\varepsilon_{T}$, impurity ionization occurs by phonon assisted tunneling where defect tunneling controls the total process. ${ }^{10}$ At larger electron energies $\varepsilon$, phonon assisted tunneling is outwighted by direct electron tunneling without involving phonons. ${ }^{10}$ For the proposed method, phonon assisted tunneling is most important.

From Eq. (1) it can be shown ${ }^{8}$ that in the regime of phonon assisted tunneling the ionization probability of deep neutral centers in an alternating electric field $\widetilde{E}(t)$ $=E \cos (\omega t)$ is given by

$$
e(E)=e(0) \exp \left[\frac{E^{2}}{\left(E_{c}^{*}\right)^{2}}\right] \text { with }\left(E_{c}^{*}\right)^{2}=\frac{3 m^{*} \hbar}{e^{2}\left(\tau_{2}^{*}\right)^{3}},
$$

where $e(0)$ is the thermal ionization probability at zero electric field, $m^{*}$ is the effective mass of the carrier, and $\tau_{2}^{*}$ is an effective time depending on the electric field frequency $\omega$ like

$$
\left(\tau_{2}^{*}\right)^{3}=\frac{3}{4 \omega^{3}}\left[\sinh \left(2 \omega \tau_{2}\right)-2 \omega \tau_{2}\right] .
$$

The tunneling times are given by ${ }^{10}$

$$
\tau_{2}=\frac{\hbar}{2 k_{B} T} \pm \tau_{1},
$$

and for the Huang-Rhys model

$$
\tau_{1}=\frac{1}{2 \omega_{\mathrm{vib}}}\left|\ln \frac{\varepsilon_{T}}{\varepsilon_{\mathrm{opt}}-\varepsilon_{T}}\right|,
$$

where $T$ is the temperature, $\omega_{\text {vib }}$ is the impurity vibration frequency, $\varepsilon_{\text {opt }}$ and $\varepsilon_{T}$ are the optical and thermal ionization energies, respectively. The plus and minus signs in Eq. (4) correspond to the adiabatic potential structures of substitutional impurities (top left in Fig. 8) and autolocalized impurities (bottom right in Fig. 8), respectively.

The electric field and temperature dependences of the ionization probability allow to deduce deep impurity parameters according to Eqs. (2)-(5). This may be done in the quasi-static limit $\omega \tau_{2} \ll 1$ most conveniently. In this limit, the effective time $\tau_{2}^{*}$ in Eq. (2) gets equal to the defect tunneling time $\tau_{2}$ yielding an ionization probability independent of the frequency $\omega$ of the alternating electric field. Thus, $\tau_{2}^{*}=\tau_{2}$ and the tunneling time $\tau_{2}$ may be determined directly from the slope of $\ln [e(E) / e(0)]$ as a function of $E^{2}$. Comparing $\tau_{2}$ with the value of $\hbar /\left(2 k_{B} T\right)$ allows to conclude on the basic structure of the defect adiabatic potentials and yields $\tau_{1}$ according to Eq. (4). From values of the tunneling time $\tau_{1}$, Eq. (5) leads to the impurity vibration frequency $\omega_{\text {vib }}$, if $\varepsilon_{\text {opt }}$ and $\varepsilon_{T}$ are known, or vice versa to the difference between optical and thermal ionization energy, if $\omega_{\text {vib }}$ and $\varepsilon_{T}$ are known.

It should be pointed out that phonon assisted tunneling, characterized by the exponential growth of the emission probability with $E^{2}$, occurs only in a limited range of electric field strength. With rising electric field strength phonon assisted tunneling proceeds into direct tunneling which shows a weaker field dependence. ${ }^{7}$ Thus, the exponential $E^{2}$ depen- 


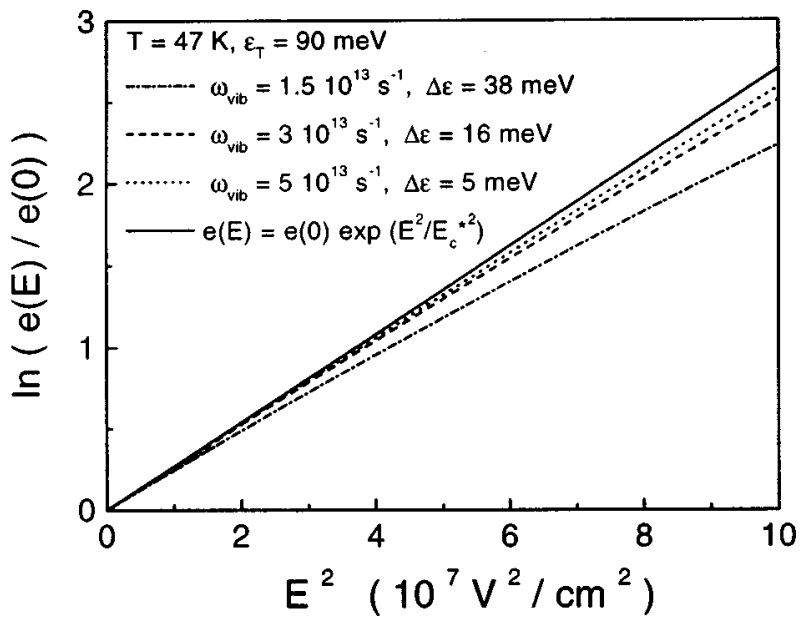

FIG. 3. Calculations for ionization of neutral impurity with $\varepsilon_{T}=90 \mathrm{meV}$ and $m^{*}=0.044 m_{e}$. The ratio of the emission probability in the electric field $e(E)$ and the thermal emission probability $e(0)$ is plotted as a function of $E^{2}$ for different values of $\omega_{\text {vib }}$ and $\Delta \varepsilon$ at $\tau_{1}=2.9 \times 10^{-14} \mathrm{~s}$.

dence of the ionization probability cannot be observed at high electric fields. At low field strength, another limit may be imposed depending on the charge state of the impurity after detachment of the carrier. In the case of neutral impurities, only tunneling causes stimulation of carrier emission by an electric field. For charged impurities, however, the lowering of the Coulomb barrier in an electric field enhances thermal emission (Poole-Frenkel effect, ${ }^{11}$ Fig. 2, broken lines). At low electric field strengths, the Poole-Frenkel effect dominates carrier emission yielding an exponential growth of the emission probability with the square root of the electric field. In the regime of phonon assisted tunneling, the impurity charge increases the transparency of the tunneling barrier leading to a constant factor in Eq. (2). ${ }^{12,13}$

Both deviations from phonon assisted tunneling can be utilized to characterize deep impurities. At low fields, the presence or missing of the Poole-Frenkel effect indicates charged or neutral impurities, respectively. At high electric field strength, the transition from phonon assisted tunneling to direct tunneling strongly depends on $\Delta \varepsilon=\varepsilon_{\text {opt }}-\varepsilon_{T}$ and $\omega_{\text {vib }}$ and allows to determine these parameters. To demonstrate this dependence, the emission probability of neutral impurities has been calculated for different combinations of these parameters at constant tunneling time $\tau_{1}$ taking into account both phonon assisted and direct tunneling. The results are plotted in Fig. 3 showing that the electric field

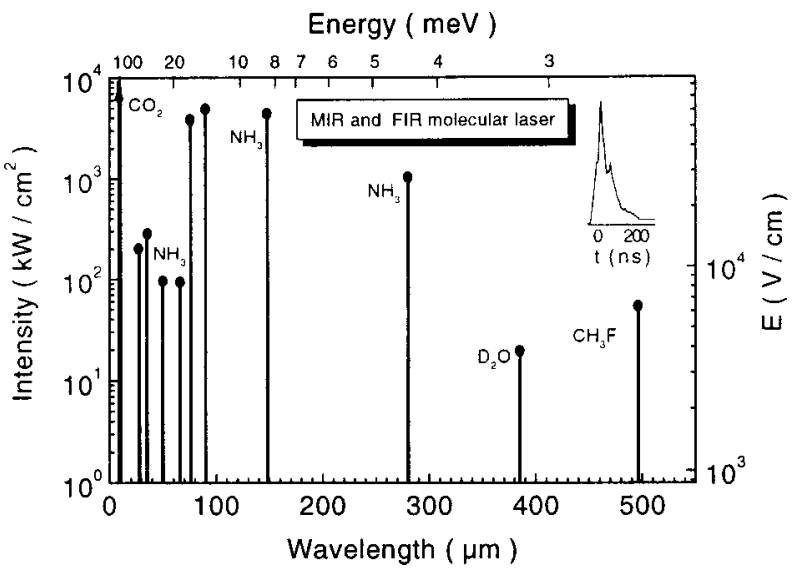

FIG. 4. Characteristics of the far-infrared laser.

strength of transition significantly shifts to higher values with rising local vibration frequency.

\section{EXPERIMENTAL SETUP}

Terahertz electric fields have been applied to semiconductor samples by illumination with an optically pumped powerful far-infrared molecular gas laser ${ }^{7}$ using $\mathrm{NH}_{3}, \mathrm{CH}_{3} \mathrm{~F}$, and $\mathrm{D}_{2} \mathrm{O}$ as laser media. The pump radiation source was a grating tunable TEA $\mathrm{CO}_{2}$ laser commercially available (URENCO-204).

The spectrum and the power of strong single laser lines in the terahertz range from 30 to $500 \mu \mathrm{m}$ used here, are presented in Fig. 4. Radiation frequencies, intensities, gain media, and pump lines are listed in Table I. The radiation pulse length varies for different lines from 10 to $100 \mathrm{~ns}$. The radiation is focused to a spot of about $1 \mathrm{~mm}^{2}$, with the maximum intensity reaching as high as $5 \mathrm{MW} / \mathrm{cm}^{2}$ corresponding in vacuum to an electric field strength of $60 \mathrm{kV} / \mathrm{cm}$. In Fig. 5 , the profile of the laser beam at $\lambda=76 \mu \mathrm{m}$ is shown.

The intensity, shape, and spatial distribution of the laser radiation were measured with fast room-temperature photodetectors operating in the far-infrared and based on the photon drag effect, ${ }^{14}$ intraband $\mu$-photoconductivity ${ }^{15}$ as well as with a Spiricon pyroelectric camera.

Two basically different kinds of deep impurity centers in semiconductors have been studied: (i) substitutional impurities with weak electron-phonon coupling and (ii) autolocalized centers with strong electron-phonon coupling ( $D X$ centers in $\mathrm{A}_{3} \mathrm{~B}_{5}$ alloys).

TABLE I. Characteristics of the far-infrared laser lines used in this work.

\begin{tabular}{cccccc}
\hline $\begin{array}{c}\text { Wavelength } \\
(\mu \mathrm{m})\end{array}$ & $\begin{array}{c}\text { Frequency } \\
\omega(\mathrm{THz})\end{array}$ & $\begin{array}{c}\text { Quantum energy } \\
\hbar \omega(\mathrm{meV})\end{array}$ & $\begin{array}{c}\text { Line of } \mathrm{CO}_{2} \\
\text { pump laser }\end{array}$ & $\begin{array}{c}\text { Max. intensity } \\
\left(\mathrm{kW} / \mathrm{cm}^{2}\right)\end{array}$ & \begin{tabular}{c} 
Medium \\
\hline 76
\end{tabular} \\
\hline 25 & 16 & $10 P(26)$ & 4000 & $\mathrm{NH}_{3}$ \\
90.5 & 21 & 14 & $9 R(16)$ & 5000 & $\mathrm{NH}_{3}$ \\
148 & 13 & 8.5 & $9 P(36)$ & 4500 & $\mathrm{NH}_{3}$ \\
250 & 7.5 & 4.9 & $9 R(26)$ & 400 & $\mathrm{CH}_{3} \mathrm{~F}$ \\
280 & 6.7 & 4.4 & $10 R(8)$ & 500 & $\mathrm{NH}_{3}$ \\
385 & 4.9 & 3.2 & $9 R(22)$ & 10 & $\mathrm{D}_{2} \mathrm{O}$ \\
496 & 3.8 & 2.5 & $9 R(20)$ & $\mathrm{CH}_{3} \mathrm{~F}$ \\
\hline \hline
\end{tabular}




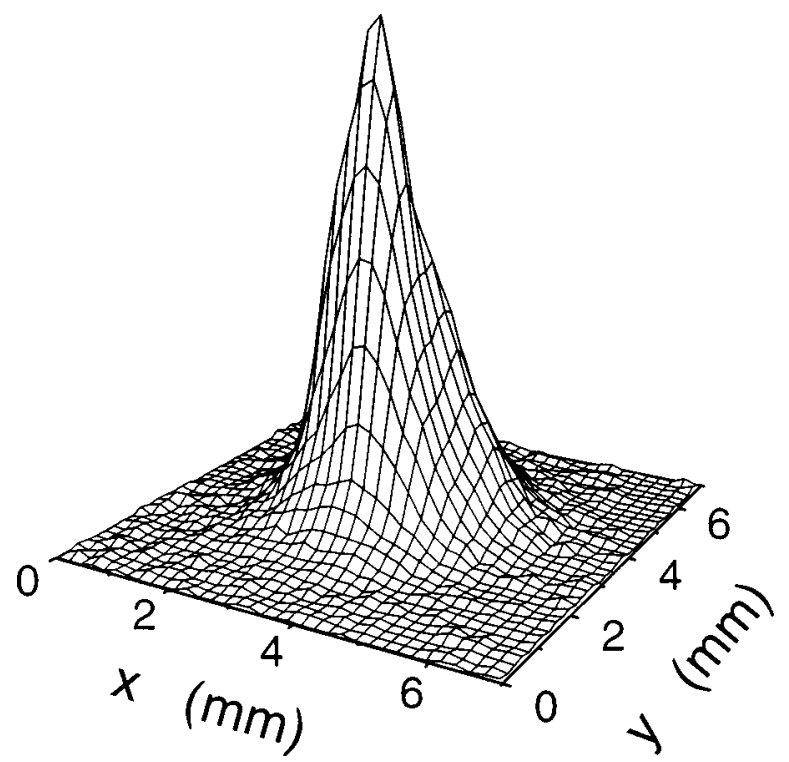

FIG. 5. Profile of the laser beam at $\lambda=76 \mu \mathrm{m}$ measured with the Spiricon camera. The resolution of the pyroelectric array is $0.1 \mathrm{~mm}$.

The measurements on substitutional impurities were carried out on $p$-type germanium doped with gold, mercury, and copper, having ionization energies of $\varepsilon_{T}=150,90$, and 40 $\mathrm{meV}$, respectively. The doping concentration was between $10^{14}$ and $10^{15} \mathrm{~cm}^{-3}$. For conditions investigated here, ionization of $\mathrm{Au}, \mathrm{Hg}$, and $\mathrm{Cu}$ impurities corresponds to a transition from a neutral ground state to a singly charged final state. Ohmic contacts were made on opposite surfaces of $2 \times 2 \times 1$ $\mathrm{mm}^{3}$ bulk samples. Measurements of ionization of $D X$ centers in $\mathrm{AlGaAs}$ (thermal ionization energy $\varepsilon_{T}=140 \mathrm{meV}$, optical ionization energy $\varepsilon_{\text {opt }}=805 \mathrm{meV}$ ) were performed on thin films of $\mathrm{Al}_{x} \mathrm{Ga}_{(1-x)}$ As $(x=0.35)$ grown by metalorganic vapor phase epitaxy (MOVPE) on semi-insulating (100) GaAs substrates. The typical layer thickness was 30 $\mu \mathrm{m}$. All layers were doped uniformly with Te. The doping concentration was $2 \times 10^{18} \mathrm{~cm}^{-3}$. The value of the dopant concentration was confirmed by both secondary ion mass spectroscopy (SIMS) and Hall effect measurements. The investigations of $D X$ centers in $\mathrm{Al}_{x} \mathrm{Ga}_{1-x} \mathrm{Sb}$ have been carried out on samples grown by the traveling heater method from $\mathrm{Sb}$ rich melts. Tellurium was added into the melt resulting in $n$-type conduction $\left(n=4 \times 10^{17} \mathrm{~cm}^{-3}\right)$. The actual sample compositions were $x=0.28$ and 0.5 . More details of the properties of the samples can be found in Ref. 7. The crystals were characterized by Hall effect, deep level transient spectroscopy (DLTS), and photocapacitance measurements. Two ohmic contact were made on opposite surfaces of $2 \times 2 \times 0.5$ $\mathrm{mm}^{3}$ bulk samples. All the essential features of the $D X$ centers, in particular, persistence photoconductivity, have been observed in both materials.

The samples were placed in a temperature-controlled optical cryostat and measurements were carried out at temperatures, where at thermal equilibrium practically all carriers are frozen out on the impurity $(T=4.2-300 \mathrm{~K}$, depending on the material). As the free carrier absorption is proportional to the free carrier concentration, which under these conditions is extremely small, even bulk samples can be illuminated ho-

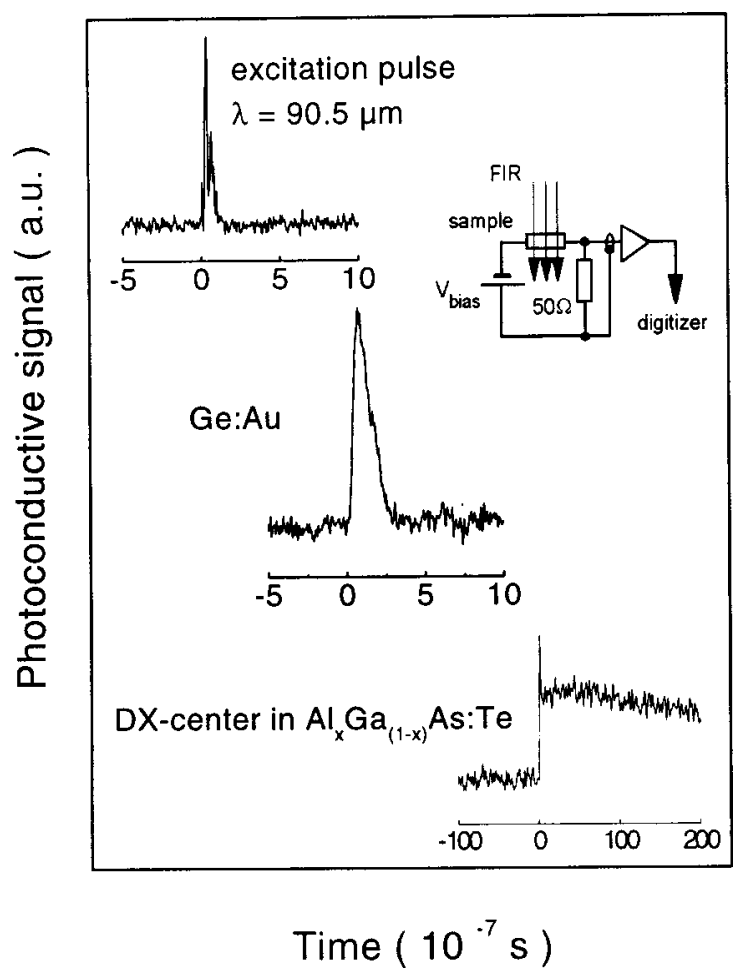

FIG. 6. Schematic representation of the experimental setup, time-resolved measurement of the excitation laser pulse, and kinetic of photoconductivity signals for two different samples. Note that for the $D X$ centers, the persistent photoconductive part of the signal has been used for analyses.

mogeneously by far-infrared radiation. The electric field strength has been calculated from the intensity inside the sample. Using calibrated teflon and polyethylene attenuators, the electric field strength has been varied. Light in the medium infrared range and in the visible was blocked by $z$-cut crystalline quartz plates and 1-mm-thick black-polyethylene sheets, respectively.

The enhancement of the thermal ionization probability due to the electric field of the radiation results in an increase of free carrier concentration. The change of the ionization probability has been detected as photoconductive signal using a standard $50 \Omega$ load resistor circuit (see inset in Fig. 6). The ratio of conductivity under illumination, $\sigma_{i}$, and dark conductivity, $\sigma_{d}$, has been determined from peak values of photoconductive signals. For laser pulses shorter than the carrier capture time, as it is the case here, $\sigma_{i} / \sigma_{d}$ is equal to $e(E) / e(0)$, where $e(E)$ is the emission rate as a function of the electric field strength $E$. Note that the FIR response in the case of $D X$ centers corresponds to the detachment of electrons from the defect yielding persistent photoconductivity.

\section{EXPERIMENTAL RESULTS AND DISCUSSION}

Ionization of deep impurities by far-infrared radiation with photon energies much smaller than the impurity binding energy has been observed in all samples. Figure 6 shows the photoconductive signal of Ge:Au and $D X$ centers in AlGaSb:Te in response to pulsed far-infrared radiation in comparison to the excitation pulse. Note that the signal observed in samples with $D X$ centers has two components, a 


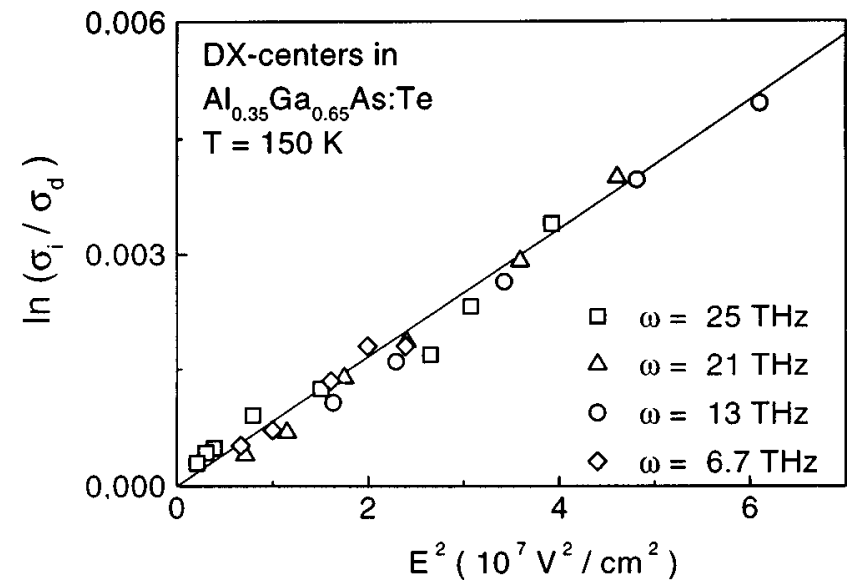

FIG. 7. Logarithm of the ionization probability, given by the ratio of the conductivity under illumination and in the dark $\ln \left(\sigma_{i} / \sigma_{d}\right)=\ln [e(E) / e(0)]$ of $D X$ centers in AlGaAs:Te plotted as a function of $E^{2}$ for $T=150 \mathrm{~K}$. Straight lines show the dependence according to Eqs. (2) and (3).

fast component reproducing the excitation pulse and a very long lasting component. The short signal pulse is caused by free carrier heating whereas the long pulse corresponds to persistent photoconductivity due to the detachment of electrons from $D X$ centers. ${ }^{16}$ For analysis of the ionization process, the persistent photoconductive signal has to be used.

The peak of the photoconductive signal corresponds to the change of the free carrier concentration at the maximum electric field strength $E$ of far-infrared radiation inside the sample. The electric field dependence of the ratio of irradiated conductivity and dark conductivity $\sigma_{i} / \sigma_{d}=e(E) / e(0)$ has been obtained by varying the laser radiation intensity. Figure 7 shows an example obtained for $D X$ centers in Tedoped AlGaAs at $T=150 \mathrm{~K}$. The logarithm of $\sigma_{i} / \sigma_{d}$ is plotted as a function of the square of the peak electric field strength of single laser pulses.

The probability of electron detachment from the deep center is independent on the radiation frequency and increases exponentially with the square of the electric field strength. $D X$ centers in AlGaAs have been chosen to demonstrate the proposed method because this material shows phonon assisted tunneling beginning with zero electric field up to rather high electric field strengths. The slope of $\ln \left(\sigma_{i} / \sigma_{d}\right)$ as a function of $E^{2}$ gives the characteristic field strength $E_{c}^{*}$ and the effective time $\tau_{2}^{*}$ can be calculated using Eq. (2). As the ionization probability is independent on the field frequency, $\tau_{2}^{*}$ is equal to the tunneling time $\tau_{2}$ at all frequencies used here.

Figure 8 shows the ionization probability of singly charged substitutional impurities mercury in germanium as a function of $E^{2}$ at $T=77 \mathrm{~K}$ in a lin-log plot. The ionization probability at high electric fields is again proportional to $\exp \left(E^{2} / E_{c}^{2}\right)$ similarly to the case of $D X$ centers in AlGaAs. However, the straight line in the $\ln [e(E) / e(0)]$ vs $E^{2}$ diagram is shifted along the ordinate to higher values. This increase of the phonon assisted tunneling probability for charged impurities is caused by the lowering of the potential barrier height due to the Coulomb potential of the impurity and is in good agreement with the semiclassical theory. At lower

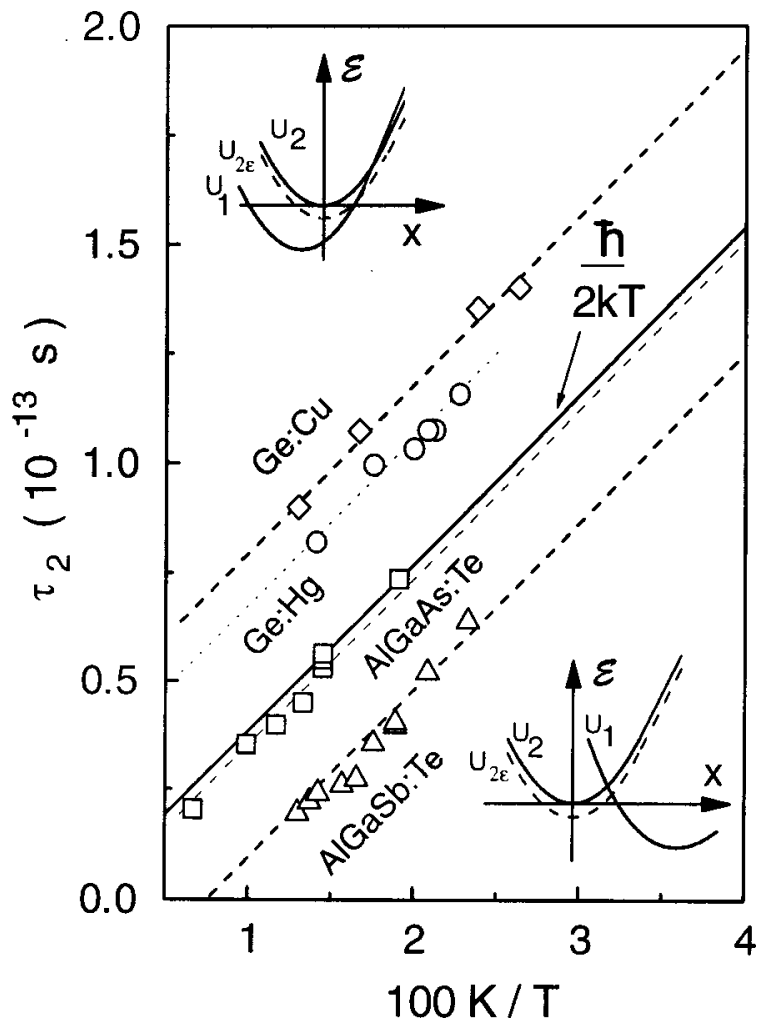

FIG. 8. Logarithm of the ionization probability $\ln \left(\sigma_{i} / \sigma_{d}\right)=\ln [e(E) / e(0)]$ of $\mathrm{Ge}: \mathrm{Hg}$ plotted as a function of $E^{2}$ for $T=77 \mathrm{~K}$. The straight line corresponds to [const $\exp \left(E^{2} / E_{c}^{2}\right)$ ]. Inset: Ionization probability as a function of the square root of the electric field to demonstrate the Poole-Frenkel effect.

fields strength, the Poole-Frenkel effect dominates carrier emission and the ionization probability exponentially grows with $\sqrt{E}$ (see inset in Fig. 8). Thus, the field dependence of the ionization probability can be used to decide the question of whether a deep impurity is charged or neutral. To determine the charge state of an impurity, two criteria can be used: (i) observation of the Poole-Frenkel effect at low fields and (ii) the shift of the straight line in the $\ln [e(E) / e(0)]$ vs $E^{2}$ diagram at higher electric fields where phonon assisted tunneling causes ionization. For the second criterion, it is essential to normalize the dependence $e(E)$ by the emission probability at zero electric field, $e(0)$. Neither the PooleFrenkel effect nor the shift have been observed for $D X$ centers in AlGaAs. Note that due to the high sensitivity of the method of photoconductivity, both phonon assisted tunneling and the Poole-Frenkel effect in alternating fields, can be measured in one experiment.

Analogous results have been obtained from several donor and acceptor doped semiconductors. In a suitable chosen temperature range where phonon assisted tunneling in the quasi-static limit applies, the ionization probability is independent of radiation frequency allowing one to determine the tunneling time $\tau_{2}$ as in the example of $D X$ centers given above. In the case of acceptors, the light hole mass should be taken to calculate $\tau_{2}$ because carriers bound to deep acceptors predominantly tunnel into the light hole band as shown in Ref. 17. Figure 9 presents the temperature dependence of the tunneling time $\tau_{2}$ obtained for various samples in the quasi-static regime $\left(\omega \tau_{2}<1\right)$ of phonon assisted tunneling. 


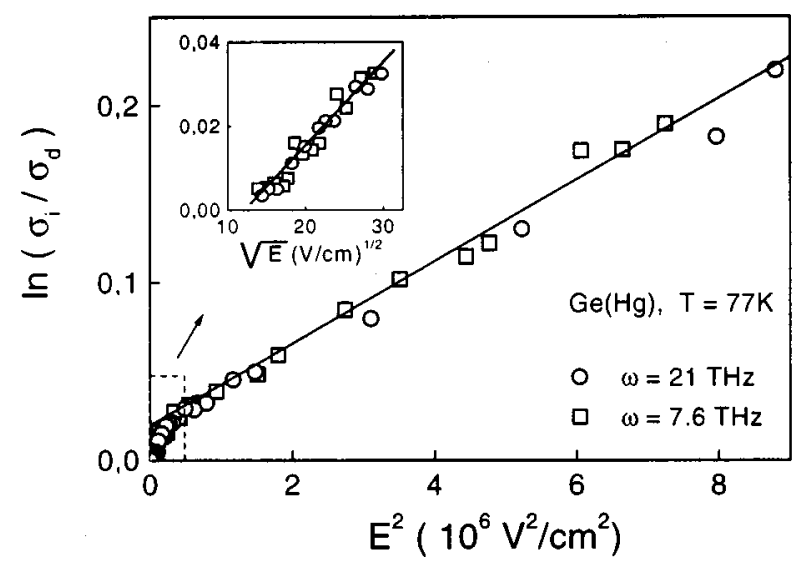

FIG. 9. Tunneling times $\tau_{2}$ as a function of $1 / T$ for different samples. The full line shows $\hbar / 2 k_{B} T$, the broken lines are plotted according to Eq. (4). The values for $\tau_{1}$ are given in Table I. Insets: Adiabatic potentials for substitutional impurities (top left) and autolocalized impurities (bottom right).

For the purpose of comparison, $\hbar /\left(2 k_{B} T\right)$ is also plotted in Fig. 9. As can be seen, $\tau_{2}$ is larger than $\hbar /\left(2 k_{B} T\right)$ for substitutional impurities and smaller than $\hbar /\left(2 k_{B} T\right)$ for the $D X$ centers. Thus, the tunneling time compared to $\hbar /\left(2 k_{B} T\right)$ reflects the basic structure of the potential barriers which is systematically distinct for both potential configurations discussed here and shown in the insets in Fig. 9.

From $\tau_{2}$ and the temperature $T$, the value of $\tau_{1}$ can be obtained using Eq. (4). The results are given in Table II. After Eq. (5), $\tau_{1}$ links the impurity thermal and optical binding energies with the local vibration frequency. As for $D X$ centers, the values of $\varepsilon_{T}$ and $\varepsilon_{\text {opt }}$ are known from literature, ${ }^{16}$ determination of $\tau_{1}$ allows to derive the local vibration frequency. For mercury in germanium, the vibration frequency is known ${ }^{18}$ as well as the thermal binding energy. ${ }^{5}$ With these data and the knowledge of $\tau_{1}$, the difference between the optical and the thermal binding energies $\Delta \varepsilon=\varepsilon_{\text {opt }}-\varepsilon_{T}$ can be determined. For substitutional impurities, $\Delta \varepsilon$ is very small because of the weak electron-phonon interaction. Indeed, this quantity has never been measured before by conventional methods. Finally, we note that these data can also be represented by the frequently used Huang-Rhys parameter $S_{\mathrm{HR}}=\Delta \varepsilon / \hbar \omega_{\mathrm{vib}}$ and the electron-phonon interaction parameter $\beta=\Delta \varepsilon / \varepsilon_{T}$. The data obtained are summarized in Table II.

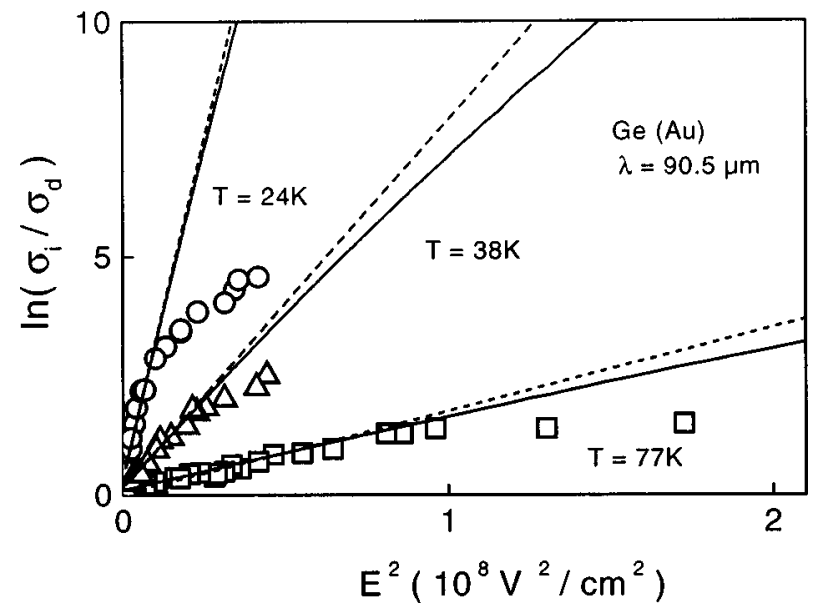

FIG. 10. Logarithm of the ionization probability $\ln \left(\sigma_{i} / \sigma_{d}\right)=\ln [e(E) / e(0)]$ of $\mathrm{Ge}:$ Au plotted as a function of $E^{2}$ for different temperatures. Solid lines are calculations after (Ref. 7) taking into account both phonon assisted and direct tunneling with parameters given in Table II. Dashed straight lines correspond to [const $\left.\exp \left(E^{2} / E_{c}^{2}\right)\right]$.

The transition from phonon assisted tunneling to direct tunneling at high electric field strength is shown in Fig. 10 for Ge:Au. The measurements and calculation after $^{10}$ were carried out at various temperatures and demonstrate that the ionization probability at high electric fields grows slower than given by the exponential $E^{2}$ relation of phonon assisted tunneling. The transition field $E_{\text {trans }}=\left(\sqrt{2 m^{*} \varepsilon_{T}}\right) /\left(\tau_{2} e\right),{ }^{7} \mathrm{de}-$ pends on the impurity binding energy and is higher for defects with bigger values for $\varepsilon_{T}$. With decreasing temperature $E_{\text {trans }}$ shifts to lower field strengths. Therefore, e.g., at $4.2 \mathrm{~K}$, the field strength where phonon assisted tunneling occurs may be so small that the exponential $E^{2}$ dependence of the ionization probability is very hard to detect with typical deep impurities. This sets a lower limit of the temperature and an upper limit of the electric field strength for the proposed method of deep impurity analysis. On the other hand, the transition from phonon assisted tunneling to direct tunneling allows one to deduce $\omega_{\text {vib }}$ and $\Delta \varepsilon=\varepsilon_{\mathrm{opt}}-\varepsilon_{T}$. These parameters can be obtained from the comparison of the theory to experimental results as shown in Fig. 10. The theory fits well the range of phonon assisted tunneling and the transition field $E_{\text {trans }}$ as a function of temperature and binding energy. The experimentally observed slower increase of the ionization probability for $E>E_{\text {trans }}$ has been discussed in Ref. 7 .

TABLE II. Parameters of samples investigated.

\begin{tabular}{|c|c|c|c|c|c|c|}
\hline & $\begin{array}{c}\varepsilon_{T} \\
(\mathrm{meV})\end{array}$ & $\begin{array}{c}\varepsilon_{\mathrm{opt}} \\
(\mathrm{meV})\end{array}$ & $\begin{array}{c}\Delta_{\varepsilon} \\
(\mathrm{meV})\end{array}$ & $\begin{array}{c}\tau_{1} \\
\left(10^{-15} \mathrm{~s}\right)\end{array}$ & $\begin{array}{c}\omega_{\mathrm{vib}} \\
\left(10^{13} \mathrm{~s}^{-1}\right)\end{array}$ & $S_{\mathrm{HR}}$ \\
\hline AlGaAs:Te & $140^{\mathrm{a}}$ & $850^{\mathrm{a}}$ & 710 & 3.3 & 25 & 4 \\
\hline AlGaSb:Te & $120^{\mathrm{a}}$ & $860^{\mathrm{a}}$ & 740 & 29 & 3.0 & 36 \\
\hline Ge:Au & $150^{\mathrm{b}}$ & 160 & 10 & 45 & 3.0 & 0.5 \\
\hline $\mathrm{Ge}: \mathrm{Hg}$ & $90^{\mathrm{b}}$ & $117,109,106$ & $27,19,16$ & 29 & $2.1,,^{\mathrm{c}} 2.7,{ }^{\mathrm{c}} 3.0$ & $1.9,1.0,0.8$ \\
\hline $\mathrm{Ge}: \mathrm{Cu}$ & $40^{\mathrm{b}}$ & $\cdots$ & $\cdots$ & 41 & $\cdots$ & $\cdots$ \\
\hline
\end{tabular}

${ }^{\mathrm{a}}$ Reference 16 .

${ }^{\mathrm{b}}$ Reference 5.

${ }^{c}$ Reference 18. 


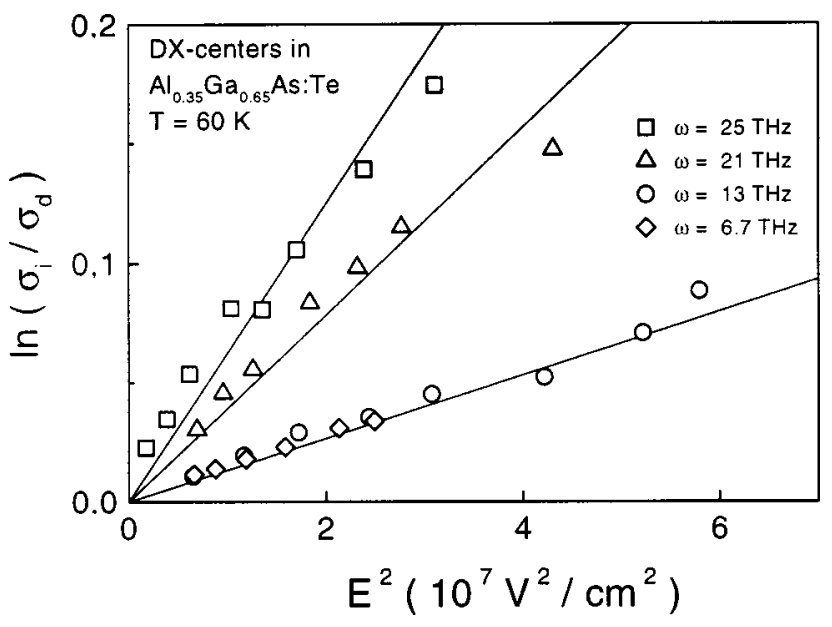

FIG. 11. Logarithm of the ionization probability $\ln \left(\sigma_{i} / \sigma_{d}\right)=\ln [e(E) / e(0)]$ of $D X$ centers in AlGaAs:Te plotted as a function of $E^{2}$ for $\omega \tau_{2} \gg 1$ at $T$ $=60 \mathrm{~K}$. Straight lines show the dependence according to Eqs. (2) and (3).

The resulting values of $\omega_{\text {vib }}$ are given in Table II and are in good agreement with previous results for $\mathrm{Hg}$ in $\mathrm{Ge}^{18}$ obtained by other methods.

Finally, we would like to emphasize that the analysis presented here is only valid in the quasi-static limit for frequencies less than $\tau_{2}^{-1}$, a condition which depends on temperature and the structure of the adiabatic potentials. At higher radiation frequencies, i.e., for $\omega \tau_{2}>1$, but with photon energies still smaller than the impurity binding energy, a strong frequency dependence of the ionization probability has been observed as shown in Fig. $11 .^{8}$ In this high frequency regime, the ionization probability of phonon assisted tunneling still depends exponentially on the square of the electric field strength but the apparent characteristic electric field $E_{c}^{*}$ cannot be used to determine the tunneling time $\tau_{2}$. The time deduced from $E_{c}^{*}$ is the effective time $\tau_{2}^{*}$ [see Eq. (3)] which strongly depends on frequency. In order to identify the quasi-static regime in high frequency measurements, $\tau_{2}^{*}$ must be compared to the inverse frequency of radiation. Only when $\omega \tau_{2}^{*} \leqslant 1$, is $\tau_{2}^{*}$ equal to the tunneling time $\tau_{2}$ and the suggested method can be applied. Typically, in the limit $\omega \tau_{2} \approx 1, \omega$ is in the range of terahertz frequencies.

\section{CONCLUSION}

Terahertz ionization of deep impurities in semiconductors has been proposed as a method for the characterization of deep impurities. The field dependence of the photoconductive signal in the range of phonon assisted tunneling allows one to determine defect tunneling times, the HuangRhys parameter, the structure of the adiabatic potentials, and the defect charge state.
All measurements carried out with terahertz radiation but in the quasi-static regime may as well be performed using static electric fields as in DLTS. However, the proposed method of impurity ionization by short far-infrared laser ase pulses permits contactless application of very strong electric fields to bulk samples as well as structured samples and, thus, helps to avoid problems associated with the onset of avalanche breakdown, current pinching, etc., which are frequently encountered when operating with strong static electric fields. The high sensitivity of the photoresponse offers a possibility of measurements over a broad field range, from tens of $\mathrm{kV} / \mathrm{cm}$ to very low field strengths where also the Poole-Frenkel effect may be detected for charged impurities.

\section{ACKNOWLEDGMENTS}

Financial support by the Deutsche Forschungsgemeinschaft, the Russian Foundation of Fundamental Investigation, the NATO Linkage Grant and the Graduiertenkolleg Regensburg is gratefully acknowledged.

${ }^{1}$ D. V. Lang, J. Appl. Phys. 45, 3014 (1974).

${ }^{2}$ S. Makram-Ebeid and M. Lannoo, Phys. Rev. B 25, 6406 (1982).

${ }^{3}$ V. Karpus and V. I. Perel, JETP Lett. 42, 497 (1985).

${ }^{4}$ P. T. Landsberg, Recombination in Semiconductors (Cambridge University Press, Cambridge, 1991).

${ }^{5}$ V. N. Abakumov, V. I. Perel, and I. N. Yassievich, Nonradiative Recombination in Semiconductors, Modern Problems in Condensed Matter Sciences Vol. 33, edited by V. M. Agranovich and A. A. Maradudin (1991).

${ }^{6}$ S. D. Ganichev, W. Prettl, and P. G. Huggard, Phys. Rev. Lett. 71, 3882 (1993).

${ }^{7}$ S. D. Ganichev, W. Prettl, and I. N. Yassievich, Phys. Solid State 39, 1703 (1997).

${ }^{8}$ S. D. Ganichev, E. Ziemann, Th. Gleim, W. Prettl, I. N. Yassievich, V. I. Perel, I. Wilke, and E. E. Haller, Phys. Rev. Lett. 80, 2409 (1998).

${ }^{9}$ M. Büttiker and R. Landauer, Phys. Rev. Lett. 49, 1739 (1982).

${ }^{10}$ V. Karpus and V. I. Perel, Zh. Éksp. Teor. Fiz. 91, 2319 (1986) [Sov. Phys. JETP 64, 1376 (1986)].

${ }^{11}$ J. Frenkel, Phys. Rev. 54, 647 (1938).

${ }^{12}$ V. N. Abakumov, V. Karpus, V. I. Perel', and I. N. Yassievich, Sov. Phys. Semicond. 22, 159 (1988).

${ }^{13}$ E. Ziemann, S. D. Ganichev, W. Prettl, A. Istratov, and E. R. Weber, in Luminescent Materials, edited by J. McKittrick, B. Di. Bartolo, and K. Mishra, Mater. Res. Soc. Symp. Proc. 560, 239 (1999).

${ }^{14}$ S. D. Ganichev, Ya. V. Terent'ev, and I. D. Yaroshetskiı̌, Sov. Tech. Phys. Lett. 11, 20 (1985).

${ }^{15}$ S. D. Ganichev, S. A. Emel'yanov, A. G. Pakhomov, Ya. V. Terent'ev, and I. D. Yaroshetskii, Sov. Tech. Phys. Lett. 11, 377 (1985).

${ }^{16}$ P. M. Mooney, J. Appl. Phys. 67, R1 (1990).

${ }^{17}$ I. A. Merkulov, A. A. Pakhomov, and I. N. Yassievich, Fiz. Tverd. Tela (Leningrad) 28, 2127 (1986).

${ }^{18}$ A. A. Pakhomov, A. F. Polupanov, V. I. Galiev, and E. Z. Imamov, Sov. Phys. Solid State 33, 416 (1991). 